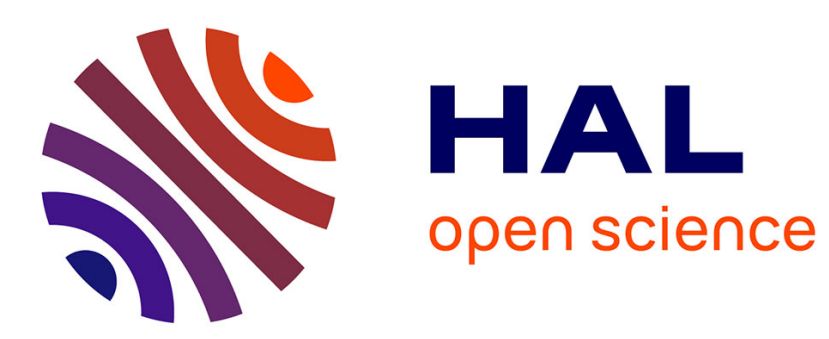

\title{
Towards an Interval-Valued Estimation of the Density
} Bilal Nehme, Olivier Strauss

\section{To cite this version:}

Bilal Nehme, Olivier Strauss. Towards an Interval-Valued Estimation of the Density. WCCI: World Congress on Computational Intelligence, 2010, Barcelona, Spain. pp.3114-3119. lirmm-00505969

\section{HAL Id: lirmm-00505969 \\ https://hal-lirmm.ccsd.cnrs.fr/lirmm-00505969}

Submitted on 27 Jul 2010

HAL is a multi-disciplinary open access archive for the deposit and dissemination of scientific research documents, whether they are published or not. The documents may come from teaching and research institutions in France or abroad, or from public or private research centers.
L'archive ouverte pluridisciplinaire HAL, est destinée au dépôt et à la diffusion de documents scientifiques de niveau recherche, publiés ou non, émanant des établissements d'enseignement et de recherche français ou étrangers, des laboratoires publics ou privés. 


\title{
Towards an interval-valued estimation of the density
}

\author{
Bilal Nehme and Olivier Strauss
}

\begin{abstract}
This paper presents a theoretical and practical novel approach for computing the probability density function underlying a set of observations. The estimator we propose is an extension of the conventional Parzen Rosenblatt method that leads to a very specific interval-valued estimation of the density. Within this approach, we make use of the convenient representation of a set of usual (summative) kernels by a maxitive kernel (i.e. a possibility distribution) to derive an exact computation with a very low complexity of an interval-valued estimation. The considered set of kernels is particularly convenient since it contains kernels having comparable shapes and bandwidth. We prove that the obtained imprecise probability density function contains a set of precise density functions estimated using the standard method with kernels belonging to the considered set.
\end{abstract}

\section{INTRODUCTION}

In the last ten years, there has been an increasing interest for imprecise probability in decision making. This framework has been mainly developed in the context of epistemic probabilities to handle the difficulty of representing ill known information by mean of precise subjective probabilities.

In the more objective context of real data processing, this framework is barely used due to the difficulty of computing or specifying the imprecise probability of any event associated to an observation process (e.g. measurement, sensing, imaging, etc.). Yet, such a knowledge could lead to new robust method for analyzing, filtering or comparing data.

In this context of real data processing, probability density function (pdf) plays a central role. Assuming a particular form of this density able, for example, comparing two sets of data via a particular distance (e.g. Mahalanobis distance) or filtering the data to remove spurious random variations. If not, this density has to be estimated from a finite sample of observations supposedly independent and identically distributed.

There are two classical ways to achieve such an estimation: parametric and nonparametric, depending on whether or not a particular model can be assumed for the density [10], [11]. Here, we concentrate on nonparametric approaches. Among the different non parametric approaches [12], the most popular is the so-called Parzen Rosenblatt method. This popularity comes from its easy computation and the easy interpretation of the density it provides [13].

In this paper, we propose an extension of the Parzen Rosenblatt approach which leads to an interval-valued probability density function [2]. Due to its construction, this interval-valued probability has a particular meaning: it is

Bilal Nehme is with the Department of Robotics, LIRMM, 161, rue Ada, 34095 Montpellier Cedex 5, France (phone: +33 467418 564; email: Bilal.Nehme@lirmm.fr).

Olivier Strauss is with the Department of Robotics, LIRMM, 161, rue Ada, 34095 Montpellier Cedex 5, France (phone: +33 467418 587; email: Olivier.Strauss@lirmm.fr). the convex set of all Parzen Rosenblatt estimations obtained with kernel having comparable shapes and comparable bandwidths. This work is restricted to symmetric bounded kernel having a first derivative, i.e. the kernel that are mostly used in this context. We also propose a practical, exact and low cost implementation of the computation of this interval-valued pdf.

This paper is organized as follows: section II introduces some necessary preliminary concepts and reformulates the Parzen Rosenblatt estimator in a way that can lead to our interval-valued estimator. In section III we show how to built our interval-valued estimator based on a particular kernel. Section IV presents the discrete algorithm leading to an exact computation of the interval valued probability in any point of a reference interval. Section V illustrates the proposed interval-valued estimation.

\section{Preliminaries}

This preliminary section aims at presenting different mathematical tools that will be used to construct the imprecise pdf estimator we propose. In the rest of the paper $\Omega$ will be the interval $\left[e_{\min }, e_{\max }\right]$ of $\mathbb{R}, \mathcal{P}(\Omega)$ the collection of all Lebesgue measurable subsets of $\Omega, \mathcal{K}(\Omega)$ the set of summative kernels in $\Omega$ and $s: \Omega \rightarrow \mathbb{R}$ a bounded $L_{1}$ function associated to a distribution in the meaning of Schwartz [9].

\section{A. Summative and maxitive kernel}

Kernels are functions from $\Omega$ to $\mathbb{R}$ that are often used in signal processing and non-parametric statistics to defining a weighted neighborhood around a location $u \in \Omega$. The distinction between summative and maxitive kernels has been introduced by [4] to handle an imprecise knowledge on the proper tool to be used in a data processing context.

A summative kernel is a positive real valued function $\kappa$ of $\Omega$, verifying the summativity property:

$$
\int_{\Omega} \kappa(u) d u=1
$$

A summative kernel $\kappa$ can be seen as a probability distribution inducing an additive confidence measure (probability) $P_{\kappa}$ defined by:

$$
\forall A \in \mathcal{P}(\Omega), P_{\kappa}(A)=\int_{A} \kappa(u) d u .
$$

A summative kernel $\kappa_{\Delta}^{x}$ can be defined by translating a generic summative kernel $\kappa$ in $x \in \Omega$ by:

$$
\forall u \in \Omega, \kappa_{\Delta}^{x}(u)=\frac{1}{\Delta} \kappa\left(\frac{u-x}{\Delta}\right),
$$


with $\Delta>0 . \Delta$ is called the bandwidth of $\kappa_{\Delta}^{x}$. By construction, $\forall u \in \Omega, \kappa(u)=\kappa_{1}^{0}(u)$.

A maxitive kernel is a real function $\pi$ from $\Omega$ to $[0,1]$, verifying the maxitivity property:

$$
\sup _{u \in \Omega} \pi(u)=1 \text {. }
$$

A maxitive kernel can be seen as a possibility distribution, inducing two dual non-additive confidence measures on $\Omega$ : a possibility measure $\Pi_{\pi}$ and a necessity measure $N_{\pi}$, defined by:

$$
\begin{array}{r}
\forall A \in \mathcal{P}(\Omega), \Pi_{\pi}(A)=\sup _{x \in A} \pi(x) \quad(\text { possibility }), \\
N_{\pi}(A)=1-\Pi_{\pi}\left(A^{c}\right) \text { (necessity), }
\end{array}
$$

with $A^{c}$ being the complementary set of $\Omega$.

A maxitive kernel $\pi_{\Delta}^{x}$ can be defined by translating a generic maxitive kernel $\pi$ in $x \in \Omega$ by:

$$
\forall u \in \Omega, \pi_{\Delta}^{x}(u)=\pi\left(\frac{u-x}{\Delta}\right),
$$

with $\Delta>0 . \Delta$ is called the bandwidth of $\pi_{\Delta}^{x}$. By construction, $\forall u \in \Omega, \pi(u)=\pi_{1}^{0}(u)$.

A maxitive kernel $\pi$ is said to dominate a summative kernel $\kappa$ [4] if the possibility measure $\Pi_{\pi}$ dominates the probability measure $P_{\kappa}$, i.e.:

$$
\forall A \in \mathcal{P}(\Omega), P_{\kappa}(A) \leq \Pi_{\pi}(A) .
$$

In that sense, a maxitive kernel defines a set of summative kernels denoted $\mathcal{M}(\pi)$ and defined by:

$$
\mathcal{M}(\pi)=\left\{\begin{array}{c}
\kappa \in \mathcal{K}(\Omega), \text { such that } \\
\forall A \in \mathcal{P}(\Omega), N_{\pi}(A) \leq P_{\kappa}(A) \leq \Pi_{\pi}(A)
\end{array}\right\} .
$$

\section{B. Derivative of a symmetric summative kernel}

In most data processing applications, the kernel used are unimodal and symmetric with a bounded support and having a first derivative. The symmetry will be an important property that will be used in the construction of our new estimator. In the rest of the paper $\mathcal{K}^{\prime}(\Omega)$ will denoted the subset of unimodal symmetric kernels of $\mathcal{K}(\Omega)$ having a bounded support and a first derivative.

Let $\kappa_{\Delta} \in \mathcal{K}^{\prime}(\Omega)$. The Jordan decomposition of its first derivative $d \kappa_{\Delta}$ is given by: $-d \kappa_{\Delta}=d \kappa_{\Delta}^{+}-d \kappa_{\Delta}^{-}$, with $d \kappa_{\Delta}^{+}=\max \left(0,-d \kappa_{\Delta}\right)$ and $d \kappa_{\Delta}^{-}=\max \left(0, d \kappa_{\Delta}\right)$.

Property 1: Let $\kappa_{\Delta} \in \mathcal{K}^{\prime}(\Omega)$. The derivative of a summative kernel $\kappa_{\Delta}$ can be written as the linear combination of two summative kernels $\eta_{\Delta}^{+}$and $\eta_{\Delta}^{-}$:

$$
\forall u \in \Omega,-d \kappa_{\Delta}(u)=a_{\Delta}^{+} \eta_{\Delta}^{+}(u)-a_{\Delta}^{-} \eta_{\Delta}^{-}(u),
$$
where $a_{\Delta}^{+}$and $a_{\Delta}^{-}$are two constants defined by $a_{\Delta}^{+}=\int_{\Omega} d \kappa_{\Delta}^{+}(u) d u$ and $a_{\Delta}^{-}=\int_{\Omega} d \kappa_{\Delta}^{-}(u) d u$.
Proof $\eta_{\Delta}^{+}$and $\eta_{\Delta}^{-}$are defined by: $\eta_{\Delta}^{+}(u)=\frac{d \kappa_{\Delta}^{+}(u)}{a_{\Delta}^{+}}$and $\eta_{\Delta}^{-}(u)=\frac{d \kappa_{\Delta}^{-}(u)}{a_{\Delta}^{-}}$.

$\eta_{\Delta}^{+}$and $\eta_{\Delta}^{-}$are positive by construction and follow the summativity condition:

$$
\int_{\Omega} \eta_{\Delta}^{+}(u) d u=\frac{1}{a_{\Delta}^{+}} \int_{\Omega} d \kappa_{\Delta}^{+}(u) d u=1,
$$

and

$$
\int_{\Omega} \eta_{\Delta}^{-}(u) d u=\frac{1}{a_{\Delta}^{-}} \int_{\Omega} d \kappa_{\Delta}^{-}(u) d u=1 .
$$

Since, by construction $d \kappa_{\Delta}^{+}=a_{\Delta}^{+} \eta_{\Delta}^{+}$and $d \kappa_{\Delta}^{-}=a_{\Delta}^{-} \eta_{\Delta}^{-}$, the expression (3) is proved.

$\kappa_{\Delta}$ being symmetric, $a_{\Delta}^{+}=a_{\Delta}^{-}=a_{\Delta}$ and the two summative kernels $\eta_{\Delta}^{+}$and $\eta_{\Delta}^{-}$can be derived from a unique summative kernel $\eta_{\Delta}: \forall u \in \Omega, \eta_{\Delta}^{+}(u)=\eta_{\Delta}\left(\frac{\Delta}{2}-u\right)$ and $\eta_{\Delta}^{-}(u)=$ $\eta_{\Delta}\left(\frac{\Delta}{2}+u\right)$. Thus the derivative of the summative kernel $\kappa_{\Delta} \in \mathcal{K}^{\prime}(\Omega)$ can be rewritten by:

$$
\forall u \in \Omega,-d \kappa_{\Delta}(u)=a_{\Delta}\left(\eta_{\Delta}\left(\frac{\Delta}{2}-u\right)-\eta_{\Delta}\left(\frac{\Delta}{2}+u\right)\right),
$$

with $a_{\Delta}=\int_{\Omega} \max \left(0,-d \kappa_{\Delta}(u)\right) d u$.

Property 2: Let $a=\int_{\Omega} \max (0,-d \kappa(u)) d u$, then, for all $\Delta>0$ :

$$
a_{\Delta}=\frac{a}{\Delta}
$$

Proof First remark that $d \kappa_{\Delta}(u)=\frac{1}{\Delta^{2}} d \kappa\left(\frac{u}{\Delta}\right)$. Thus

$$
\begin{aligned}
a_{\Delta} & =\int_{\Omega} \max \left(0,-d \kappa_{\Delta}(u)\right) d u=\int_{\Omega} \max \left(0,-\frac{1}{\Delta^{2}} d \kappa\left(\frac{u}{\Delta}\right)\right) d u \\
& =\Delta \int_{\Omega} \max \left(0,-\frac{1}{\Delta^{2}} d \kappa(u)\right) d u=\frac{1}{\Delta} \int_{\Omega} \max (0,-d \kappa(u)) d u \\
& =\frac{a}{\Delta}
\end{aligned}
$$

\section{Derivative in the sense of distributions}

Convolution is a mathematical way of combining two functions to form a third function. The convolution of function $s$ by a summative kernel $\kappa$, denoted $\widehat{s}_{\kappa}$, is given by:

$$
\widehat{s}_{\kappa}(x)=\int_{\Omega} s(u) \kappa(x-u) d u=\int_{\Omega} s(u) \kappa^{x}(u) d u=\left\langle s, \kappa^{x}\right\rangle,
$$

$\kappa^{x}$ being the function $\kappa$ translated in $x$, and $\langle.,$.$\rangle being$ the dot product defined for $L_{1}$ functions. If the summative kernel $\kappa$ is derivable, it can be seen as a test function. It is thus possible to link $d s$, the derivative of $s$ in the sense of 
distribution, to $d \kappa$, the derivative of $\kappa$ in the sense of function by:

$$
\begin{aligned}
\left\langle d s, \kappa^{x}\right\rangle & =\int_{\Omega} d s(u) \kappa^{x}(u) d u=-\int_{\Omega} s(u) d \kappa^{x}(u) d u \\
& =-\left\langle s, d \kappa^{x}\right\rangle
\end{aligned}
$$

\section{From precise to imprecise kernel based expectation}

In this section, we introduce summative and maxitive based expectation operators of a function $s$. An expectation based on a summative kernel $\kappa$ coincides with the usual expectation based on the probability measure $P_{\kappa}$ associated with $\kappa$. It is defined by:

$$
\mathbb{E}_{\kappa}(s)=\int_{\Omega} s d P_{\kappa}=\int_{\Omega} s(u) \kappa(u) d u .
$$

The expectation based on a maxitive kernel $\pi$ has been introduced in [3]. It uses the conventional extension of the expectation operator, called the Choquet integral [1], and has an interval-valued output. It is defined by:

$$
\underline{\mathbb{E}}_{\pi}(s)=\left[\underline{\mathbb{E}}_{\pi}(s), \overline{\mathbb{E}}_{\pi}(s)\right],
$$

with $\underline{\mathbb{E}}_{\pi}(s)=\mathbb{C}_{N_{\pi}}(s)$ and $\overline{\mathbb{E}}_{\pi}(s)=\mathbb{C}_{\Pi_{\pi}}(s), \mathbb{C}_{\Pi_{\pi}}(s)$ (resp. $\mathbb{C}_{N_{\pi}}(s)$ ) being the Choquet integral of $s$ with respect to the possibility measure $\Pi_{\pi}$ (resp. the necessity measure $N_{\pi}$ ). This imprecise valued expectation operator respects the following interesting properties derived from the domination properties defined in section II-A :

$$
\forall y \in \underline{\underline{\mathbb{E}}}_{\pi}(s), \exists \kappa \in \mathcal{M}(\pi) / \mathbb{E}_{\kappa}(s)=y,
$$

and

$$
\forall \kappa \in \mathcal{M}(\pi), \mathbb{E}_{\kappa}(s) \in \underline{\overline{\mathbb{E}}}_{\pi}(s) .
$$

This property has been proved in [3].

\section{E. Reformulation of the Parzen Rosenblatt estimator}

In this section, we propose a reformulation of the classical Parzen Rosenblatt estimator. This reformulation will be the basis of the new operator we propose.

Let $\left(x_{1}, \ldots, x_{n}\right)$ be a sample of $n$ i.i.d. observations drawn from a population having an unknown pdf $f$. The Parzen Rosenblatt kernel estimate [6], [8] of $f$ in every point $x \in \Omega$ is given by:

$$
\widehat{f}_{\kappa_{\Delta}}^{n}(x)=\frac{1}{n \Delta} \sum_{i=1}^{n} \kappa\left(\frac{x-x_{i}}{\Delta}\right)=\frac{1}{n \Delta} \sum_{i=1}^{n} \kappa_{\Delta}^{x}\left(x_{i}\right) .
$$

Property 3: The estimation $\widehat{f}_{\kappa_{\Delta}}^{n}$ in every point $x \in \Omega$ can be rewritten as the dot product kernels $\kappa_{\Delta}^{x}$ with the empirical measure $e_{n}$ :

$$
\widehat{f}_{\kappa_{\Delta}}^{n}(x)=\left\langle e_{n}, \kappa_{\Delta}^{x}\right\rangle,
$$

with $e_{n}=\frac{1}{n} \sum_{i=1}^{n} \delta^{x_{i}}$ and $\delta^{x_{i}}$ is the impulse Dirac translated in $x_{i}$.
Proof According to (6), we have, for all $x \in \Omega$ :

$$
\begin{aligned}
\left\langle e_{n}, \kappa_{\Delta}^{x}\right\rangle & =\int_{\Omega} \frac{1}{n} \sum_{i=1}^{n} \delta^{x_{i}}(u) \kappa_{\Delta}^{x}(u) d u, \\
& =\frac{1}{n} \sum_{i=1}^{n} \int_{\Omega} \delta^{x_{i}}(u) \kappa_{\Delta}^{x}(u) d u, \\
& =\frac{1}{n} \sum_{i=1}^{n} \kappa_{\Delta}^{x}\left(x_{i}\right)=\widehat{f}_{\kappa_{\Delta}}^{n}(x) .
\end{aligned}
$$

The estimation $\widehat{f}_{\kappa_{\Delta}}^{n}$ in every point $x \in \Omega$ can be interpreted as the precise expectation of the empirical distribution $e_{n}$ according to a neighborhood of $x$ defined by the summative kernel $\kappa_{\Delta}$ :

$$
\widehat{f}_{\kappa_{\Delta}}^{n}(x)=\mathbb{E}_{\kappa_{\Delta}^{x}}\left(e_{n}\right)=\left\langle e_{n}, \kappa_{\Delta}^{x}\right\rangle .
$$

Let $E_{n}$ be the empirical distribution function defined by:

$$
\forall x \in \Omega, \quad E_{n}(x)=\frac{1}{n} \sum_{i=1}^{n} H\left(x-x_{i}\right),
$$

$H$ being the Heaviside function defined by: $H(x)=1$ if $x \geq 0$ and 0 elsewhere.

$e_{n}$ being the derivative of $E_{n}$ in the sense of distributions, the Parzen Rosenblatt estimator can be rewritten, for all $x \in \Omega$, as:

$$
\widehat{f}_{\kappa_{\Delta}}^{n}(x)=\left\langle e_{n}, \kappa_{\Delta}^{x}\right\rangle=\left\langle d E_{n}, \kappa_{\Delta}^{x}\right\rangle=\left\langle E_{n},-d \kappa_{\Delta}^{x}\right\rangle .
$$

Theorem 1: Let $\kappa_{\Delta} \in \mathcal{K}^{\prime}(\Omega)$, whose Jordan decomposition of its first derivative $d \kappa_{\Delta}$ is: $\forall u \in \Omega,-d \kappa_{\Delta}(u)=$ $a_{\Delta}\left(\eta_{\Delta}\left(\frac{\Delta}{2}-u\right)-\eta_{\Delta}\left(\frac{\Delta}{2}+u\right)\right)$, with $a_{\Delta} \in \mathbb{R}^{+}$and $\eta_{\Delta} \in$ $\mathcal{K}(\Omega)$, then, for all $x \in \Omega$ :

$$
\widehat{f}_{\kappa_{\Delta}}^{n}(x)=a_{\Delta} \mathbb{E}_{\eta_{\Delta}}\left(E_{n}^{x-}-E_{n}^{x+}\right),
$$

with $\forall u \in \Omega, E_{n}^{x-}(u)=E_{n}\left(x+\frac{\Delta}{2}-u\right)$ and $E_{n}^{x+}(u)=$ $E_{n}\left(x-\frac{\Delta}{2}+u\right)$.

Proof According to (15), we have, for all $x \in \mathbb{R}$ :

$$
\begin{aligned}
\widehat{f}_{\kappa_{\Delta}}^{n}(x) & =-\mathbb{E}_{d \kappa_{\Delta}^{x}}\left(E_{n}\right), \\
& =\int_{-\infty}^{+\infty}-d \kappa_{\Delta}(u-x) E_{n}(u) d u \\
& =a_{\Delta}\left(\int_{-\infty}^{+\infty} \eta_{\Delta}\left(\frac{\Delta}{2}-u+x\right) E_{n}(u) d u\right. \\
& \left.-\int_{-\infty}^{+\infty} \eta_{\Delta}\left(\frac{\Delta}{2}+u-x\right) E_{n}(u) d u\right), \\
& =a_{\Delta}\left(\int_{-\infty}^{+\infty} \eta_{\Delta}(v) E_{n}\left(x+\frac{\Delta}{2}-v\right) d v\right. \\
& \left.-\int_{-\infty}^{+\infty} \eta_{\Delta}(v) E_{n}\left(x-\frac{\Delta}{2}+v\right) d v\right), \\
= & a_{\Delta}\left(\int_{-\infty}^{+\infty} \eta_{\Delta}(v)\left(E_{n}^{x-}(v)-E_{n}^{x+}(v)\right) d v\right), \\
= & a_{\Delta} \mathbb{E}_{\eta_{\Delta}}\left(E_{n}^{x-}-E_{n}^{x+}\right) .
\end{aligned}
$$




\section{IMPRECISE ESTIMATION OF DENSITY}

In this section, we propose an interval-valued estimation of $f$, the pdf underlying a set of observations $\left(x_{1}, \ldots, x_{n}\right)$. This new operator is based on defining a particular set of summative kernels and using the know domination properties of the maxitive kernels to derive a very specific interval-valued estimation. This interval-valued estimation contains all the Parzen Rosenblatt estimators obtained with kernels belonging to this set. This set of summative kernel is defined as follows:

$\mathcal{D}(\pi, a, \Delta)=\left\{\begin{array}{c}\nu \in \mathcal{K}^{\prime}(\Omega), \exists \xi \in \mathcal{M}\left(\pi_{\Delta}\right), \text { such that } \\ -d \nu(u)=a_{\Delta}\left(\xi\left(\frac{\Delta}{2}-u\right)-\xi\left(\frac{\Delta}{2}+u\right)\right)\end{array}\right\}$,

where $a \in \mathbb{R}^{+}\left(a_{\Delta}=\frac{a}{\Delta}\right), \pi$ is a bounded maxitive kernel and $\Delta \in \mathbb{R}^{*+}$ is a bandwidth. Choosing randomly $\pi, a$ and $\Delta$ can lead to an empty set $\mathcal{D}(\pi, a, \Delta)$.

If $\pi, a$ and $\Delta$ are chosen such that there is a summative kernel $\kappa_{\Delta} \in \mathcal{K}^{\prime}(\Omega)$ such that: $\forall u \in \Omega,-d \kappa_{\Delta}(u)=$ $a_{\Delta}\left(\eta_{\Delta}\left(\frac{\Delta}{2}-u\right)-\eta_{\Delta}\left(\frac{\Delta}{2}+u\right)\right)$, with $\eta_{\Delta} \in \mathcal{K}(\Omega), a_{\Delta} \in \mathbb{R}^{+}$ and for $\pi$ being a maxitive kernel that dominates $\eta$, then, the subset $\mathcal{D}(\pi, a, \Delta)$ is not empty since it contains $\kappa_{\Delta}$ by construction. Moreover, this set contains kernels whose bandwidth cannot exceed $\Delta$ (since the bandwidth of its two derivatives are limited by $\frac{\Delta}{2}$ ) and cannot be lower that $\frac{\Delta}{2}$. This last configuration corresponds to the uniform kernel of length $\frac{\Delta}{2}$.

Let $\kappa_{\Delta} \in \mathcal{K}^{\prime}(\Omega)$ be a summative kernel, whose Jordan decomposition of its first derivative is: $\forall u \in \Omega,-d \kappa_{\Delta}(u)=$ $a_{\Delta}\left(\eta_{\Delta}\left(\frac{\Delta}{2}-u\right)-\eta_{\Delta}\left(\frac{\Delta}{2}+u\right)\right)$, with $a_{\Delta} \in \mathbb{R}^{+}$and $\eta_{\Delta} \in$ $\mathcal{K}(\Omega)$. Let $\pi$ be the most specific maxitive kernel dominating $\eta[4]$.

Definition 1: The interval-valued estimation of the pdf, whose empirical distribution is $E_{n}$, is defined, for all $x \in \Omega$ by:

$$
\underline{f}_{\left[\kappa_{\Delta}\right]}^{n}(x)=a_{\Delta} \overline{\mathbb{E}}_{\pi_{\Delta}}\left(E_{n}^{x-}-E_{n}^{x+}\right) .
$$

The specificity of the interval-valued estimation, defined by (16), is due to the following property:

Property 4: Let $\bar{f}_{\left[\kappa_{\Delta}\right]}^{n}$ be the interval-valued estimation, then, for all $x \in \Omega$ :

$$
\forall \varphi \in \mathcal{D}(\pi, a, \Delta), \widehat{f}_{\varphi}^{n}(x) \in \bar{f}_{\left[\kappa_{\Delta}\right]}^{n}(x) .
$$

Proof According to (10), we have:

$$
\forall \xi \in \mathcal{M}\left(\pi_{\Delta}\right), \mathbb{E}_{\xi}\left(E_{n}^{x-}-E_{n}^{x+}\right) \in \underline{\mathbb{E}}_{\pi_{\Delta}}\left(E_{n}^{x-}-E_{n}^{x+}\right),
$$

multiplying by $a_{\Delta}$ the expression (17) is proved.

The reverse inclusion defined by:

$\forall y \in \bar{f}_{\left[\kappa_{\Delta}\right]}^{n}(x), \exists \xi \in \mathcal{M}\left(\pi_{\Delta}\right)$ and $\varphi \in \mathcal{K}^{\prime}(\Omega)$ such that $-d \varphi(u)=a_{\Delta}\left(\xi\left(\frac{\Delta}{2}-u\right)-\xi\left(\frac{\Delta}{2}+u\right)\right)$ and $y=\widehat{f}_{\varphi}^{n}(x)$, is not proved from now.

\section{Computation AND Algorithm}

This section aims at proposing a practical and efficient computation of the interval-valued estimation proposed in section III. Such a computation is based on the fact that $E_{n}$ is a step function whose step positions are known. It leads to an exact computation of $\bar{f}_{\left[\kappa_{\Delta}\right]}^{n}(x)$ for any $x \in \Omega$.

Let $\Delta E_{n}^{x}=E_{n}^{x-}-E_{n}^{x+}$. According to (8), the intervalvalued estimation of $f$, given by (16), can be rewritten by:

$$
\begin{aligned}
\underline{f}_{\left[\kappa_{\Delta}\right]}^{n}(x) & =a_{\Delta} \underline{\mathbb{E}}_{\pi_{\Delta}}\left(\Delta E_{n}^{x}\right), \\
& =a_{\Delta}\left[\mathbb{C}_{N_{\pi_{\Delta}}}\left(\Delta E_{n}^{x}\right), \mathbb{C}_{\Pi_{\pi_{\Delta}}}\left(\Delta E_{n}^{x}\right)\right],
\end{aligned}
$$

with $a_{\Delta} \in \mathbb{R}^{+}, \mathbb{C}_{\Pi_{\pi_{\Delta}}}\left(\Delta E_{n}^{x}\right)$ (resp. $\mathbb{C}_{N_{\pi_{\Delta}}}\left(\Delta E_{n}^{x}\right)$ ) being the Choquet integral of $\Delta E_{n}^{x}$ with respect to the possibility measure $\Pi_{\pi_{\Delta}}$ (resp. the necessity measure $N_{\pi_{\Delta}}$ ).

Since $E_{n}$ is a step function, $\Delta E_{n}^{x}$ is also step function defined by:

$$
\begin{aligned}
\forall u \in \Omega, \Delta E_{n}^{x}(u) & =\frac{1}{n} \sum_{i=1}^{n}\left(H\left(x-x_{i}+\frac{\Delta}{2}-u\right)\right. \\
& \left.-H\left(x-x_{i}-\frac{\Delta}{2}+u\right)\right), \\
& =\frac{1}{n} \sum_{i=1}^{n}\left\{\begin{aligned}
-1 & \text { if } u \geq x-x_{i}+\frac{\Delta}{2}, \\
+1 & \text { if } u \leq x_{i}-x+\frac{\Delta}{2}, \\
0 & \text { otherwise. }
\end{aligned}\right.
\end{aligned}
$$

By construction $\Delta E_{n}^{x}$ has at most $(2 n+1)$ different values. Let $\left\{w_{i}^{x}\right\}_{i \in\{0, \ldots, 2 n+1\}}$ be the set of $(2 n+2)$ value derived from the set of observations, by:

$$
w_{i}^{x}= \begin{cases}\frac{\Delta}{2}+x_{i}-x, & \text { if } i \in\{1, \ldots, n\}, \\ \frac{\Delta}{2}+x-x_{i-n}, & \text { if } i \in\{n+1, \ldots, 2 n\},\end{cases}
$$

with $w_{0}^{x}=e_{\min }$ and $w_{2 n+1}^{x}=e_{\max }$.

Let $\Theta=\{0, \ldots, 2 n\}$ and (.) be the permutation that sorts the $w_{i}^{x}$ in ascending order, i.e. $w_{(0)}^{x} \leq w_{(2)}^{x} \leq \ldots w_{(2 n+1)}^{x}$. From the set of $w_{i}^{x}$, we can define $(2 n+1)$ intervals $W_{i}^{x}$ by: $W_{i}^{x}=\left\{\left[w_{(i)}^{x}, w_{(i+1)}^{x}[\}_{i \in \Theta}\right.\right.$. By construction, $\Delta E_{n}^{x}$ is constant on each interval $W_{i}^{x}$.

Let $\alpha_{n}^{i}$ be the constant value of $\Delta E_{n}^{x}$ on $W_{i}^{x}$ defined by:

$$
\alpha_{n}^{i}=\frac{1}{n} \sum_{k=1}^{n}\left\{\begin{aligned}
-1 & \text { if } c_{i} \geq x-x_{k}+\frac{\Delta}{2}, \\
+1 & \text { if } c_{i} \leq x_{k}-x+\frac{\Delta}{2}, \\
0 & \text { otherwise. }
\end{aligned}\right.
$$

$c_{i}$ being the median value of each $W_{i}^{x}$.

Then, $\Delta E_{n}^{x}$ can be rewritten in:

$$
\forall u \in \Omega, \quad \Delta E_{n}^{x}(u)=\sum_{i=0}^{2 n} \alpha_{n}^{i} 1_{W_{i}^{x}}(u),
$$

$\mathbb{1}_{A}$ being the indicator function of $A \in \mathcal{P}(\Omega)$ defined by: $\mathbb{1}_{A}(u)=1$ if $u \in A$ and 0 elsewhere.

The computation of the interval-valued estimation, defined by (18), involves two Choquet integrals. Due to the stepwise nature of the function to be integrated, the continuous Choquet integral can be computed by a discrete Choquet integral involving a possibility distribution on the $\left\{W_{i}^{x}\right\}_{i \in \Theta}$. Let $\mu_{\Delta}$ be the discrete possibility distribution induced on $\Theta$ 
by the continuous possibility distribution $\pi$ on $\Omega$ and defined by:

$$
\forall k \in \Theta, \mu_{k_{\Delta}}=\Pi_{\pi_{\Delta}}\left(W_{k}^{x}\right) .
$$

Let $\alpha_{n}=\left\{\alpha_{n}^{i}\right\}_{i \in \Theta}$ be the $(2 n+1)$ real values of $\Delta E_{n}^{x}$, defined by (20). Let (.) be the permutation that sorts the $\alpha_{n}^{i}$ in ascending order, i.e. $\alpha_{n}^{(0)} \leq \alpha_{n}^{(1)} \leq \ldots \alpha_{n}^{(2 n)}$. Let $\left\{A_{(i)}\right\}_{i \in \Theta}$ be the $(2 n+1)$ subsets of $\Theta$ defined by: $A_{(i)}=$ $\{(i), \ldots,(2 n)\}$.

The two continuous Choquet integrals of $\Delta E_{n}^{x}$ with respect to the continuous possibility measure $\Pi_{\pi_{\Delta}}$ (resp. necessity measure $N_{\pi_{\Delta}}$ ) on $\Omega$ can be computed by the two discrete Choquet integrals of $\alpha_{n}$ with respect to the discrete possibility measure $\Pi_{\mu_{\Delta}}$ (resp. necessity measure $N_{\mu_{\Delta}}$ ) on $\Theta$. Such a computation is given by:

$\mathbb{C}_{\Pi_{\pi_{\Delta}}}\left(\Delta E_{n}^{x}\right)=\mathbb{C}_{\Pi_{\mu_{\Delta}}}\left(\alpha_{n}\right)=\sum_{i=1}^{2 n}\left(\alpha_{n}^{(i)}-\alpha_{n}^{(i-1)}\right) \Pi_{\mu_{\Delta}}\left(A_{(i)}\right)$,

and

$\mathbb{C}_{N_{\pi_{\Delta}}}\left(\Delta E_{n}^{x}\right)=\mathbb{C}_{N_{\mu_{\Delta}}}\left(\alpha_{n}\right)=\sum_{i=1}^{2 n}\left(\alpha_{n}^{(i)}-\alpha_{n}^{(i-1)}\right) N_{\mu_{\Delta}}\left(A_{(i)}\right)$

Computing the interval valued $\underline{f}_{\left[\kappa_{\Delta}\right]}^{n}$ on a location $x \in \Omega$ can be decomposed in 5 steps:

Step 1. Compute the set of $(2 n+2)$ values $\left\{w_{i}^{x}\right\}_{i \in\{0, \ldots, 2 n+1\}}$ by means of (19).

Step 2. Sort the $\left\{w_{i}^{x}\right\}$ and compute the $(2 n+1)$ intervals $W_{i}^{x}$ by: $W_{i}^{x}=\left\{\left[w_{(i)}^{x}, w_{(i+1)}^{x}[\}_{i \in\{0, \ldots, 2 n\}}\right.\right.$.

Step 3. Compute the constant values $\left\{\alpha_{n}^{i}\right\}_{i \in\{0, \ldots, 2 n\}}$ by using (20).

Step 4. Compute the two discrete Choquet integrals $\mathbb{C}_{\Pi_{\mu_{\Delta}}}\left(\alpha_{n}\right)$ and $\mathbb{C}_{N_{\mu_{\Delta}}}\left(\alpha_{n}\right)$ by means of (23) and (24).

Step 5. Multiply the result obtained in step 4 by $a_{\Delta}$.

The estimation of the pdf is usually computed on $p$ regularly spaced points of $\Omega$. Let $\left\{y_{j}\right\}_{j \in\{1, \ldots, p\}}$ be those points, the following algorithm uses the procedure described previously to compute the $p$ interval valued estimations of the pdf: $\left\{\left[\underline{f}_{\left[\kappa_{\Delta}\right]}^{n}\left(y_{j}\right), \bar{f}_{\left[\kappa_{\Delta}\right]}^{n}\left(y_{j}\right)\right]\right\}_{j \in\{1, \ldots, p\}}$.

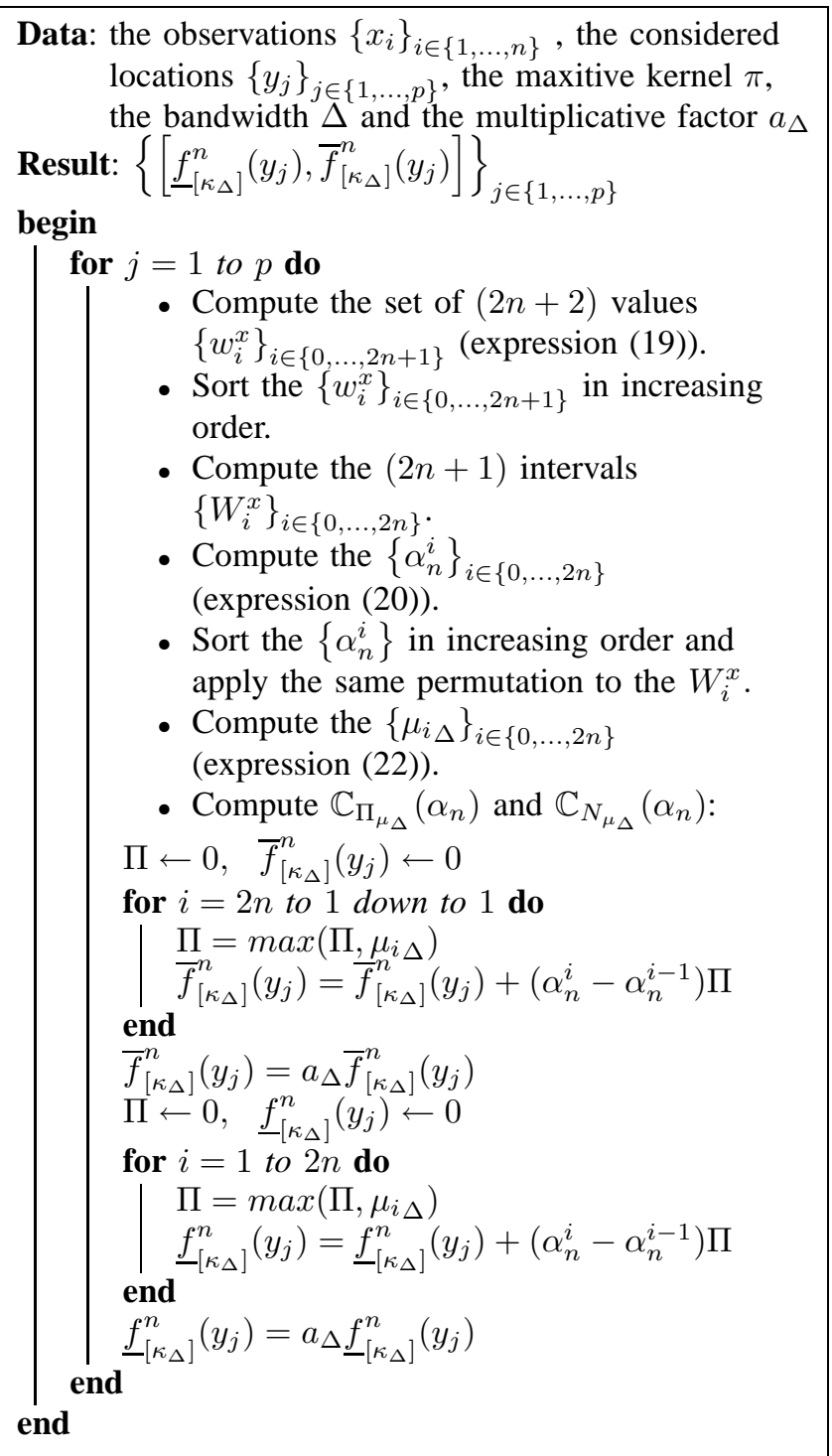

Algorithm 1: Computation of $\left\{\bar{f}_{\left[\kappa_{\Delta}\right]}^{n}\left(y_{j}\right)\right\}_{j \in\{1, \ldots, p\}}$.

\section{EXPERIMENT}

In this experiment we propose to illustrate Property 4 defined in section III. Property 4 says that, if $\kappa_{\Delta}$ is a symmetric summative kernel with a bounded support and having a first derivative, then, the precise estimate $\hat{f}_{\kappa_{\Delta}}^{n}$, defined by (11), is included in the imprecise estimate $\underline{f}_{\left[\kappa_{\Delta}\right]}^{\kappa_{\Delta}}$ defined by (16).

In this experiment, the symmetric summative kernel we use is defined on $\Omega$ by:

$$
\forall x \in \Omega, \kappa_{\Delta}(x)=\frac{1}{2 \Delta}\left(1+\cos \left(\frac{|x| \pi}{\Delta}\right)\right) \mathbb{1}_{[-\Delta, \Delta]}(x) .
$$

The summative kernel involved in the Jordan decomposition (4) of the derivative of $\kappa_{\Delta}$ is given by:

$$
\forall x \in \Omega, \eta_{\Delta}(x)=\frac{\pi}{2 \Delta}\left(\cos \left(\frac{|x| \pi}{\Delta}\right)\right) \mathbb{1}_{\left[-\frac{\Delta}{2}, \frac{\Delta}{2}\right]}(x) .
$$

The constant value $a_{\Delta}$ involved in this decomposition is equal to $\frac{1}{\Delta}$. We then construct the most specific maxitive 
kernel dominating $\eta_{\Delta}[4]$ by:

$$
\forall x \in \Omega, \pi_{\Delta}(x)=\left(1-\sin \left(\frac{|x| \pi}{\Delta}\right)\right) \mathbb{1}_{\left[-\frac{\Delta}{2}, \frac{\Delta}{2}\right]}(x) .
$$

To achieve this experiment, we have drawn 1000 observations $\left\{x_{i}\right\}_{i \in\{1, \ldots, 1000\}}$ from a simulated process whose pdf is a mixture of two Gaussian distributions with mean 3 (resp. 8) and variance 1 (resp. 4 ). The reference interval $\Omega=[-5,20]$ is divided in 500 equally spaced samples $\left\{y_{i}\right\}_{i \in\{1, \ldots, 500\}}$. The value of the bandwidth $\Delta$ has been set to 1 since it seems to be adapted to this pdf with this number of observations.

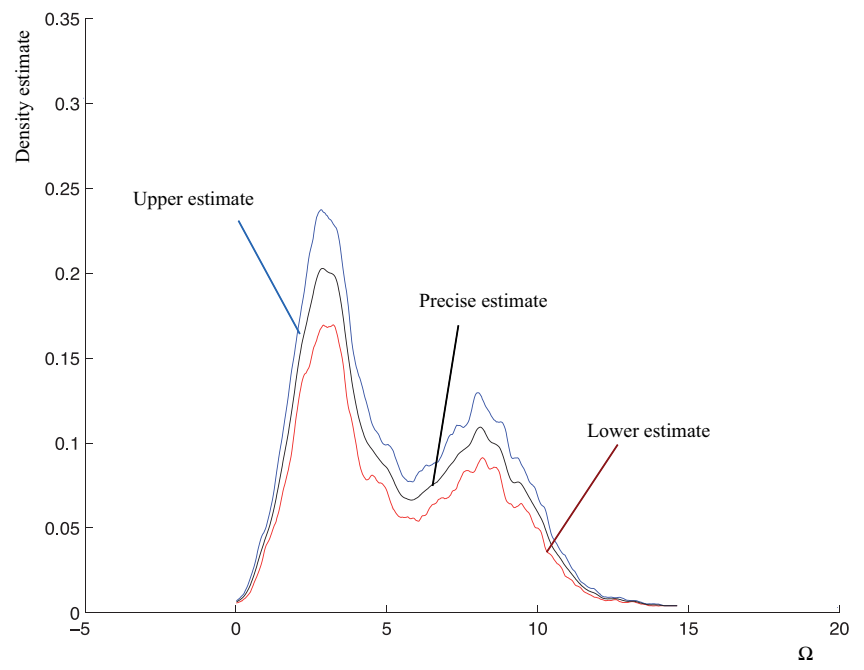

Fig. 1. Superposition, for $\Delta=1$, of the specific imprecise estimate $\underline{f}_{\left[\kappa_{\Delta}\right]}^{n}$ and the precise estimate $\hat{f}_{\kappa_{\Delta}}^{n}$.

Fig. 1 shows the superposition of the precise estimate $\hat{f}_{\kappa_{\Delta}}^{n}$ and the specific imprecise estimate $\bar{f}_{\left[\kappa_{\Delta}\right]}^{n}$. The precise estimate is plotted in black, the lower (resp. upper) estimate is plotted in red (resp. blue). As can be seen in Fig. 1, for each value $\left\{y_{i}\right\}_{i \in\{1, \ldots, 500\}}$ of the reference interval, $\hat{f}_{\kappa_{\Delta}}^{n}\left(y_{i}\right) \in \bar{f}_{\left[\kappa_{\Delta}\right]}^{n}\left(y_{i}\right)$.

\section{CONCLUSION}

In this paper, we have presented a new way for estimating the pdf underlying a finite set of observations with a practical implementation. One of the main characteristic of this estimator is that its output is interval-valued. By construction, this interval-valued probability density estimation is nothing else but the convex set of all the densities that should have been obtained by using the conventional Parzen-Rosenblatt technique with different kernels belonging to a particular set. This set of kernel has a certain interest, since it is the set of all the derivable kernels having a symmetric bounded shape with a bandwidth belonging to a limited interval. Derivable symmetric bounded kernels are among the most used in this context. Thus this set can be instrumental to represent an imprecise knowledge on the shape or bandwidth that has to be used to compute a precise density. Moreover, as shown recently [5] this kind of method can lead to a quantification of the identification error.

A great amount of work remains to answer different questions:

- does this estimator converge and in which sense? For example, how many samples are needed to guarantee a $90 \%$ inclusion of the true density in the estimated imprecise density? Does this interval-valued estimate converge to a precise-valued estimate ? From our first attempt it seems that this property is not true,

- does the median of this interval valued pdf have a particular meaning? Does it converge to the true density when the bandwidth tends to 0 and the number of observations tends to infinity?

- can the imprecision of each interval-valued pdf estimation can be considered as a quantification of the estimation error? Can this approach being compared with the traditional method consisting in estimating confidence intervals of a precise-valued pdf estimation [7]?

- is it possible to define a robust distance value between the pdf underlying two sets of observations based on this imprecise estimate?

Future work should also concentrate on practical use of this new density estimate.

\section{REFERENCES}

[1] G. Choquet. Theory of capacities. Ann. Inst. Fourier, 5:131-295, 1953.

[2] L. de Campos, J. Huete, and S. Moral. Probability intervals: a tool for uncertain reasoning. international Journal of Uncertainty, Fuzziness and Knowledge-Based Systems, 2:167-196, 1994.

[3] K. Loquin and O. Strauss. Imprecise functional estimation: the cumulative distribution case. In SMPS Soft Methods in Probability and Statistics, pages 175-182, Toulouse, France, Sept. 2008.

[4] K. Loquin and O. Strauss. On the granularity of summative kernels. Fuzzy sets and systems, 159:1952-1972, 2008.

[5] K. Loquin and O. Strauss. Noise quantization via possibilistic filtering. In ISIPTA'09: Proceedings of the Sixth International Symposium on Imprecise Probability: Theories and Applications, pages 297-306, Durham, UK, July 2009.

[6] E. Parzen. On estimation of a probability density function and mode. The Annals of Mathematical Statistics, 33:1065-1076, 1962.

[7] D. Picard and K. Tribouley. Adaptive confidence interval for pointwise curve estimation. Annals of statistics, 28:298 - 335, 2000.

[8] M. Rosenblatt. Remarks on some nonparametric estimates of a density function. The Annals of Mathematical Statistics, 27:832-837, 1956.

[9] L. Schwartz. Théorie des distributions. Hermann, Paris, 1950.

[10] D. W. Scott. Multivariate Density Estimation:Theory, Practice, and Visualization. Wiley Interscience, New York, 1992.

[11] B. Silvermann. Density Estimation for Statistics and Data Analysis. Chapman and Hall, London, 1986.

[12] A. Tsybakov. Introduction to Nonparametric Estimation. Springer science \& business media, New York, 2009.

[13] E. Wegman. Nonparametric probability density estimation. II: A comparison of density estimation methods. Statistical computation and simulation, 1:225-245, 1972. 